\title{
Successful management of recurrent puerperal uterine inversion
}

\section{Bindu Nambisan*}

Department of Obstetrics and Gynecology, Government Medical College, Thiruvananthapuram, Kerala, India

Received: 11 August 2016

Accepted: 06 September 2016

\section{*Correspondence:}

Dr. Bindu Nambisan,

E-mail: bindu.nambisan1971@gmail.com

Copyright: ( ) the author(s), publisher and licensee Medip Academy. This is an open-access article distributed under the terms of the Creative Commons Attribution Non-Commercial License, which permits unrestricted non-commercial use, distribution, and reproduction in any medium, provided the original work is properly cited.

\begin{abstract}
The puerperal uterine inversion is a rare and severe complication occurring in the third stage of labour. The exact mechanisms are unclear. However, extrinsic factors such as prolonged labour, umbilical cord traction, oxytocic use etc. have been mentioned. Other intrinsic factors such as primiparity, uterine hypotonia, different placental localizations, fundal location of a myoma or short umbilical cord have also been reported. The diagnosis of uterine inversion is mainly made on the basis of clinical symptoms which include haemorrhage, shock and a strong pelvic pain. The immediate treatment of the uterine inversion is required. A case of 23 years old, second gravida with one previous spontaneous first trimester abortion, who had a full term normal vaginal delivery but while trying to deliver the placenta after confirmation of placental separation clinically, uterine inversion was diagnosed immediately and manual repositioning of uterus was done under general anaesthesia. On the 6 th post natal day, during the routine postnatal rounds, uterus was not palpable per abdomen and a local examination revealed a mass at the introitus. A diagnosis of grade 3 sub-acute inversion was made and she was taken up for exploratory laparotomy. Reinsertion was done according to the Huntington technique by placing clamps on the round ligament, near its insertion on the uterus, and applying traction upwards while the assistant exerted traction on the contra lateral way through the vagina. As persistent atonicity and diffuse oozing was noted multiple Cho sutures were put over the uterus. Patient had an uneventful postnatal period. This is a rare scenario where the same patient had an acute inversion initially followed by sub-acute inversion.
\end{abstract}

Keywords: Hypovolemic shock, Obstetric emergency, Prolapse, Uterine bleeding, Uterine inversion

\section{INTRODUCTION}

Uterine inversion is a rare obstetric emergency whose incidence varies from 1 in 2000 to 1 in every $50,000 .{ }^{1}$ The mechanisms are not completely known. When not immediately identified, the massive and often underestimated blood loss can lead to hypovolemic shock. $^{2}$ The diagnosis of the uterine inversion is mainly based on clinical symptoms which includes haemorrhage, shock and a strong pelvic pain. The best management options for this condition are also not fully known .Literature search has revealed several management options used by different surgical teams on a case to case basis. Several therapeutic strategies are described in the literature, including drugs, manual manoeuvres and surgical interventions. ${ }^{3}$ The immediate treatment of the uterine inversion is the desired management. Two classifications of uterine inversion are generally used. The first classification is based on the severity of inversion. ${ }^{4}$ In first-degree inversion, the inverted wall extends to but not through the cervix. In second-degree inversion, the inverted wall protrudes through the cervix but remains within the vagina. In third-degree inversion, the inverted fundus extends outside the vagina. In fourthdegree or total inversion, the vagina and uterus are inverted. ${ }^{5}$ The second classification is according to the delay between the delivery and the diagnosis of the uterine inversion and is classified as acute, sub-acute and chronic variety. The acute inversions are those that arise immediately or within 24 hours after delivery. The sub- 
acute inversion include those that occur between the first 24 hours and within four weeks and chronic inversions are those that occur more than four weeks after delivery. ${ }^{6}$ The prevalence of each class of inversion is $83.4 \%$, $2.62 \%$ and $13.9 \%$ respectively. ${ }^{7}$

\section{CASE REPORT}

23 years old second gravida with one previous first trimester abortion and excellent dates was admitted for safe confinement. She gave history of regular antenatal check-ups and all antenatal investigations and ultrasound scans were normal. She was induced with two doses of prostaglandin E 2 gel (indication for induction was past date, unfavourable cervix) and pitocin acceleration was given to augment labour. She had a full term normal delivery and delivered a baby of weight $2.8 \mathrm{~kg}$ and good Apgar score. While trying to deliver out the placenta uterine inversion occurred. Manual repositioning of uterus was done under GA. She was put on broad spectrum antibiotics for 7 days. On the $6^{\text {th }}$ postnatal day, during the routine postnatal rounds, uterus was not palpable per abdomen and patient complained of mass coming per vaginum. Examination revealed an inverted uterine fundus at the introitus and a diagnosis of subacute inversion was made. She was taken up for exploratory laparotomy under general anaesthesia. The intra-operative findings revealed dimpling at the uterine fundus with the fallopian tube and round ligaments buried inside. Medial end of the round ligament was pulled from above by the surgeon using atraumatic forceps and simultaneously the prolapsed mass was pushed from below by an assistant with her hand inside the vagina and repositioning done. Uterus showed persistent atonicity and diffuse oozing. Multiple Cho sutures were put over the uterus and $\mathrm{B} / \mathrm{L}$ uterine arteries and utero-ovarian anastomosis were ligated prophylactically. Oxytocics were administered and vagina packed with roller gauze in an attempt to prevent another inversion due to uterine flaccidity. Pack was removed the next day and patient had an uneventful period thereafter and she returned for postnatal check-up after 6 weeks. Uterus was well involuted and she was asymptomatic.

\section{DISCUSSION}

The reported incidence of uterine inversion varies widely due to variation in reporting and also of obstetric practices. The combination of a fundally implanted placenta, flaccidity of the myometrium around the implantation site, and a dilated cervix is thought to predispose to puerperal inversion. In this patient, it can be logically assumed that she had an intrinsic faccidity of the myometrium considering the fact that she had a recurrent inversion. Following uterine inversion, prompt treatment of any associated haemorrhage and shock is vital in limiting morbidity and avoiding mortality.

Once the diagnosis is established, hypotension and hypovolemia require aggressive fluid resuscitation in accordance with the STAR protocol (i.e., shock, treat aggressively, and repair) Optimal treatment for inversion employs parenteral tocolytics to permit prompt uterine replacement, followed by uterotonics to maintain normal uterine positioning. ${ }^{5}$ Nonsurgical techniques like correction of partial inversions using hydrostatic pressure by instilling warm saline rapidly into the vagina was described by $\mathrm{O}$ ' Sullivan. The fluid progressively distends the vaginal wall and then forces the fundus upward to resume its original position. Some clinicians favour a trial of this procedure in selected cases because of its simplicity. With the advent of potent tocolytics, the technique of manual replacement has been greatly simplified. A variant of the manipulations, originally described by Johnson in 1949, is now the recommended procedure.

In a manual replacement operation, the operator's open hand is placed in the vagina and the inverted fundus is positioned in the palm of the hand. The uterus is then forcefully lifted upward and anteriorly through the pelvis into the abdominal cavity in the pelvic curve to the level of the umbilicus. This causes the uterine ligaments to stretch. The cervix opens, the uterus inverts, and the fundus is promptly replaced in its usual anatomic position. The uterus is held in place for several minutes to permit the supporting ligaments to return to their original state and to allow uterotonics to firm the myometrium. To facilitate the uterine replacement, terbutaline, magnesium sulfate, and nitro-glycerine all have been successfully employed as uterine relaxants, with and without concomitant general anesthesia. Following successful replacement, several doses of uterotonics are administered to avoid reinversion and to firm the myometrium.

If 2 or more attempts at manual replacement fail, surgery is indicated. If the placenta has not separated before the replacement operation is attempted, the best plan is to leave it undisturbed until the patient is in the operating room. Immediate placental removal without replacement simply increases blood loss. Rarely, in the uncommon event of a placenta accreta/increta/percreta, removal proves difficult or impossible and the attempt markedly increases blood loss. ${ }^{8}$ Because the diagnosis of abnormal placental adherence cannot be established until removal is attempted, the best plan is to wait till the patient is shifted to operating theatre room rather than to attempt immediate and perhaps incomplete removal. Failure of non-surgical techniques necessitates surgical techniques and the abdominal approach is favoured. This technique was originally described by Huntington. ${ }^{6,7}$

A laparotomy is performed, and the round ligaments are identified. Following administration of a tocolytic, the surgeon applies gentle upward traction on the round ligaments. The process is repeated with the operator progressively pulling the round ligaments up into the peritoneal cavity until the fundus is completely restored to its normal configuration. Assistance by a second 
operator, applying upward pressure from below, facilitates this procedure. As the uterus begins to revert, the lower segment is squeezed to accelerate the replacement. As in the manual replacement technique, uterotonics are administered once the uterus has reverted to its normal position. If the Huntington operation fails, the more extensive Haultain procedure is required at laparotomy. ${ }^{6}$

In this technique, a posterior, longitudinal hysterotomy incision is performed. This acutely widens the lower uterine segment and facilitates uterine replacement. In this surgical approach, the posterior uterine wall is incised to avoid inadvertent injury to the bladder, with upward traction on the round ligaments. After repositioning, uterine atony is common. Reinversion may occur. The prompt administration of 15-methyl F2 alpha prostaglandin, high-dose oxytocin, or misoprostol per rectum is recommended. If magnesium sulfate was administered as a tocolytic, calcium gluconate can be administered to reverse the tocolytic effect. Administration of a course of antibiotics has been advocated when inversion occurs, based on the idea that the necessary manipulations predispose to infection.

Funding: No funding sources Conflict of interest: None declared

Ethical approval: Not required

\section{REFERENCES}

1. Dwivedi S, Gupta N, Mishra A, Pande S, Lal P. Uterine inversion: a shocking aftermath of mismanaged third stage of labour. Int $\mathrm{J}$ Reprod Contracept Obstet Gynecol. 2013;2(3):292-5.

2. Hostetler DR, Bosworth MF. Uterine inversion: a life threatening obstetric emergency. J Am Board Fam Pract. 2000;13:120-3.

3. Watson P, Besch N, Bowes A. Management of acute and sub-acute puerperal inversion of the uterus Obstet Gynecol. 1980;55:12-6.

4. Wendel PJ, Cox SM. Emergent Obstetric management of uterine inversion. Obstet Gynecol Clin North Am. 1995; 22:261-74.

5. Repke J, Ramin S, Barss V. Puerperal uterine inversion. Available from: http://www.UpToDate.com. 2013.

6. Gerber S. uterine inversion. Rev Med Suisse Romande. 1996;116:277-83.

7. Dali SM, Rajbhandadari S, Shrestha S. Puerperal inversion ofthe uterus in Nepal: case reports and review of literature. J Obstet Gynaecol. Res 1997;23:319-25.

8. Witteveen T, Van Stralen G, Zwart J, Van Roosmalen J. Puerperal uterine inversion in the Netherlands: a nationwide cohort study. Acta Obstet Gynecol Scand. 2013;92:334.

9. Acién P, Acién M, Ferrer MS. Complex malformations of the female genital tract. New types and revision of classification. Hum Reprod. 2004; 19:2377-84.

10. Farber M, Marchant DJ. Congenital absence of the uterine cervix. American journal of obstetrics and gynecology. 1975;121(3):414-7.

11. Creighton SM, Davies MC, Cutner A. Laparoscopic management of cervical agenesis. Fertil Steril. 2006;85:1510.e13-5.

Cite this article as: Nambisan B. Successful management of recurrent puerperal uterine inversion. Int J Reprod Contracept Obstet Gynecol

2016;5:3619-21. 